CRYSTALLOGRAPHIC COMMUNICATIONS

ISSN 2056-9890

Received 20 July 2016

Accepted 2 August 2016

Edited by W. T. A. Harrison, University of Aberdeen, Scotland

Keywords: crystal structure; arylboronic acid; hydrogen-bond network; polymorph.

CCDC references: $1497347 ; 1497346$

Supporting information: this article has supporting information at journals.iucr.org/e

\section{Two forms of (naphthalen-1-yl)boronic acid}

\author{
Kayleigh Bemisderfer and Alexander Y. Nazarenko*
}

Chemistry Department, SUNY Buffalo State, 1300 Elmwood Ave, Buffalo, NY 14222, USA. *Correspondence e-mail: nazareay@buffalostate.edu

Two polymorphs of the title compound, $\mathrm{C}_{10} \mathrm{H}_{9} \mathrm{BO}_{2}$, were prepared by recystallization from different solvents at room temperature. Both forms demonstrate nearly identical molecular structures with all naphthalene group atoms located in one plane and all boronic acid atoms in another: the dihedral angles between these planes are 39.88 (5) and $40.15(5)^{\circ}$ for the two asymmetric molecules of the orthorhombic form and $40.60(3)^{\circ}$ for the single asymmetric molecule in the monoclinic form. In each extended structure, molecules form dimers, connected via two $\mathrm{O}-\mathrm{H} \cdots \mathrm{O}$ hydrogen bonds. The dimers are connected by further $\mathrm{O}-\mathrm{H} \cdots \mathrm{O}$ hydrogen bonds, forming layered networks in the (001) plane and the (100) plane in the orthorhombic and monoclinic forms, respectively. The resulting layers are practically identical in both forms. However, these layers are shifted along the [010] axis in the two forms, resulting in a slightly more effective packing for monoclinic structure (packing index = 0.692) compared to the orthorhombic form (0.688).

\section{Chemical context}

Naphthalene boronic acids ( $\alpha$ - and $\beta$-) were first synthesized by Michaelis (1894) along with other arylboronic acid by reaction of diarylmercury with boron trichloride with subsequent hydrolysis. A more practical procedure (König \& Scharrnbeck, 1930) included the reaction of naphthylmagnesium bromide with tri-(isobutyl)borate. In both cases, the existence of two different forms of title compound was suggested, one forming plate-like crystals and another one forming needles.<smiles>OB(O)c1cccc2ccccc12</smiles>

These compounds were originally investigated because of their potential in biochemistry (König \& Scharrnbeck, 1930; Gao et al., 2003; Hall, 2011) and later as reactants in the Suzuki reaction (Hall, 2011). 1-Naphthalene boronic acid is now commercially available and was the source for this study.

\section{Synthesis and crystallization}

A sample of 1-naphthalene boronic acid was purchased from Aldrich. Its FTIR spectrum coincided with that reported by 


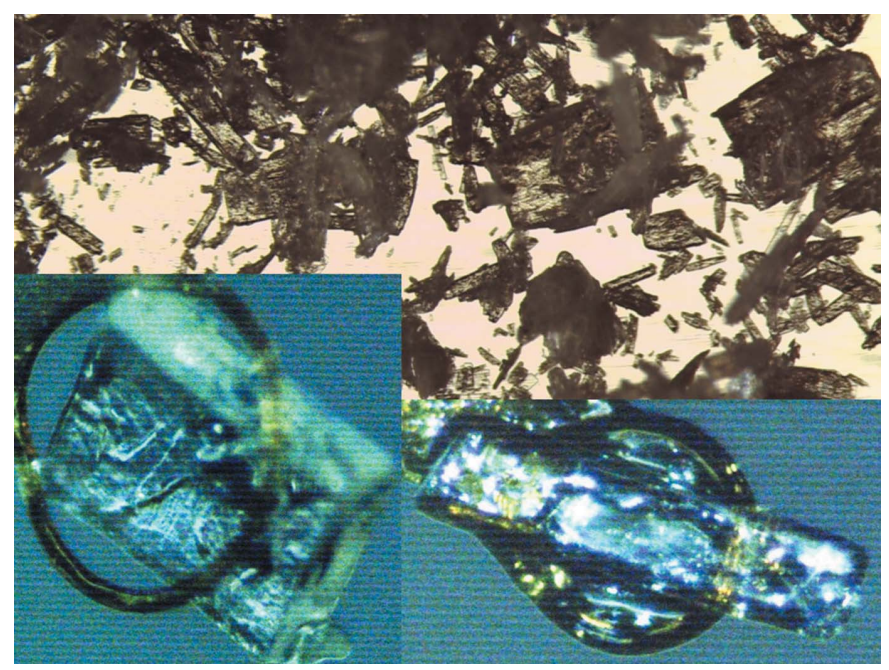

Figure 1

Crystals of the different polymorphs in starting material (view area $1 \times$ $2 \mathrm{~mm}$ ). Plate (left): orthorhombic. Needle (right): monoclinic.

the manufacturer. Under the microscope, a number of relatively large (up to $0.5 \mathrm{~mm}$ ) crystals were visible, some of them suitable for single crystal X-ray data collection (Fig. 1). Experimental data revealed an orthorhombic structure for the plate-shaped crystals. Recrystallization from hot water yielded very thin plates. This polycrystalline sample showed a powder diffractogram that was slightly different from the raw material and the calculated pattern of the orthorhombic form. Attempts at slow crystallization from ethanol and toluene solution resulted in larger and better shaped crystals, some of which were orthorhombic plates and other were visibly nonorthorhombic needles (Fig. 1). Several such crystals were tested: here we report the best data for both the orthorhombic and monoclinic forms.

\section{Structural commentary}

The molecules of naphthalene boronic acid in both crystal structures (Figs. 2 and 3) have the usual bond distances and angles. There is one molecule in the asymmetric unit of the monoclinic structure. In the non-centrosymmetric orthorhombic structure, the two molecules in the asymmetric unit

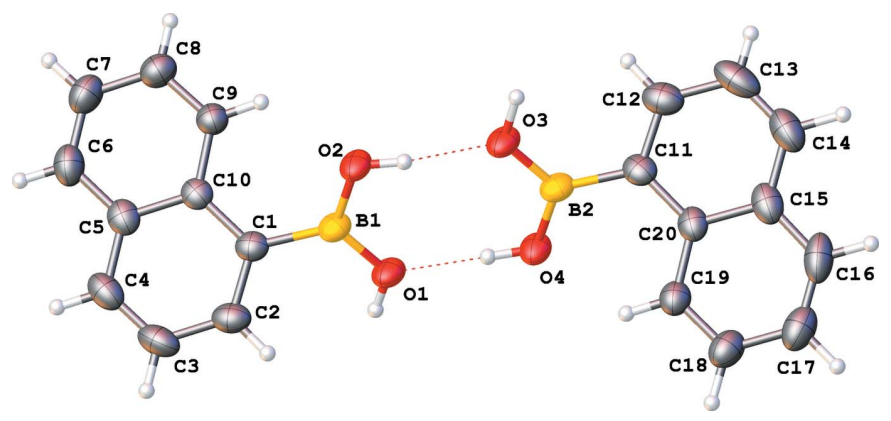

Figure 2

Numbering scheme of the title compound with $50 \%$ probability displacement ellipsoids (orthorhombic polymorph).

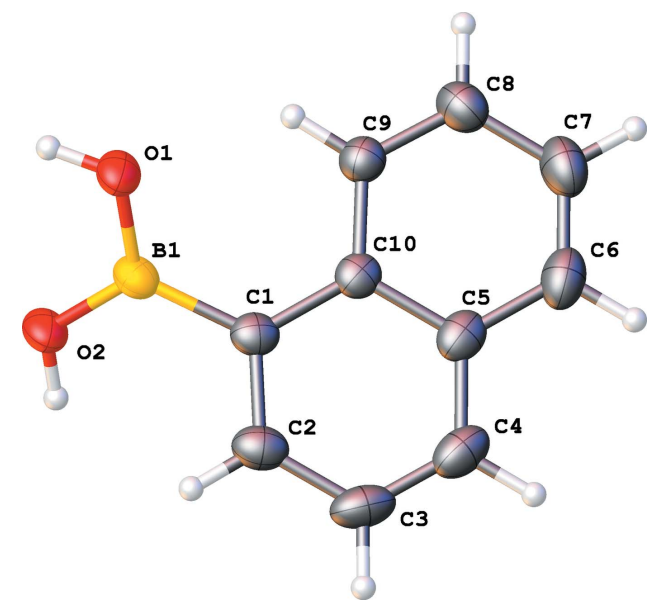

Figure 3

Numbering scheme of the title compound with $50 \%$ probability displacement ellipsoids (monoclinic polymorph).

have very similar structures: they almost coincide (after inversion for one of them) with each other as well, as with the unique molecule from the monoclinic structure (Fig. 4).

In the monoclinic structure, the mean plane of the naphthalene fragment is tilted from plane of boron and two oxygen atoms with an angle of $40.60(3)^{\circ}$. The boron atom deviates by 0.0449 (16) $\AA$ from the mean plane of the naphthalene ring system.

In the orthorhombic structure, there are two independent molecules. When superimposed, the angle between the mean planes of the naphthalene ring systems is only $0.88(6)^{\circ}$. Two boron atoms and four oxygen atoms are located at another plane together with adjacent hydrogen atoms. These planes are tilted to a similar extent to the monoclinic structure, with dihedral angles to the mean plane of each naphthalene group of $39.88(5)$ and $40.15(5)^{\circ}$ [mean tilt $=39.83(5)^{\circ}$ ]. These numbers differ from those for the monoclinic form by less than $1^{\circ}$.

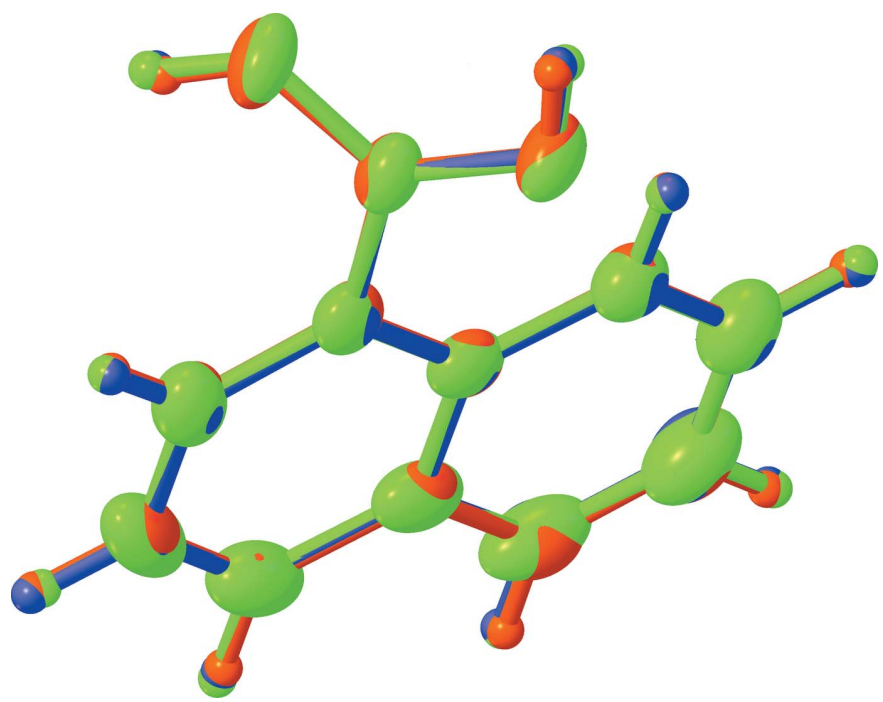

Figure 4

Overlay of the two polymorph molecules (red \& green - orthorhombic, blue - monoclinic) with appropriate inversion. 


\section{Supramolecular features}

In both forms, pairs of molecules are connected through a pair of $\mathrm{O}-\mathrm{H} \cdots \mathrm{O}$ hydrogen bonds (Tables 1 and 2) into dimers. There is also an intramolecular $\mathrm{C}-\mathrm{H} \cdots \mathrm{O}$ contact. The dimers are further connected via $\mathrm{O}-\mathrm{H} \cdots \mathrm{O}$ hydrogen bonds, forming a layered network in plane (001) and in plane (100) in the orthorhombic and monoclinic forms, respectively (Figs. 5 and $6)$. The resulting layers are practically identical in both forms (compare Figs. 7 and 8, Figs. 9 and 10).

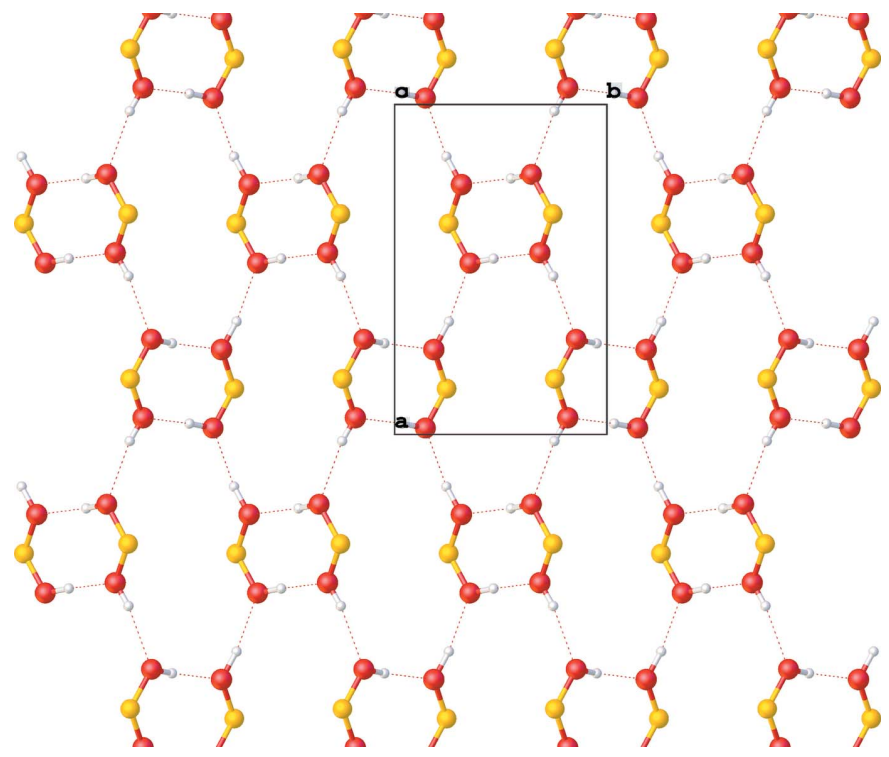

Figure 5

Layered network of hydrogen bonds in the orthorhombic form. View is along the [001] axis, only boronic acid groups are shown.
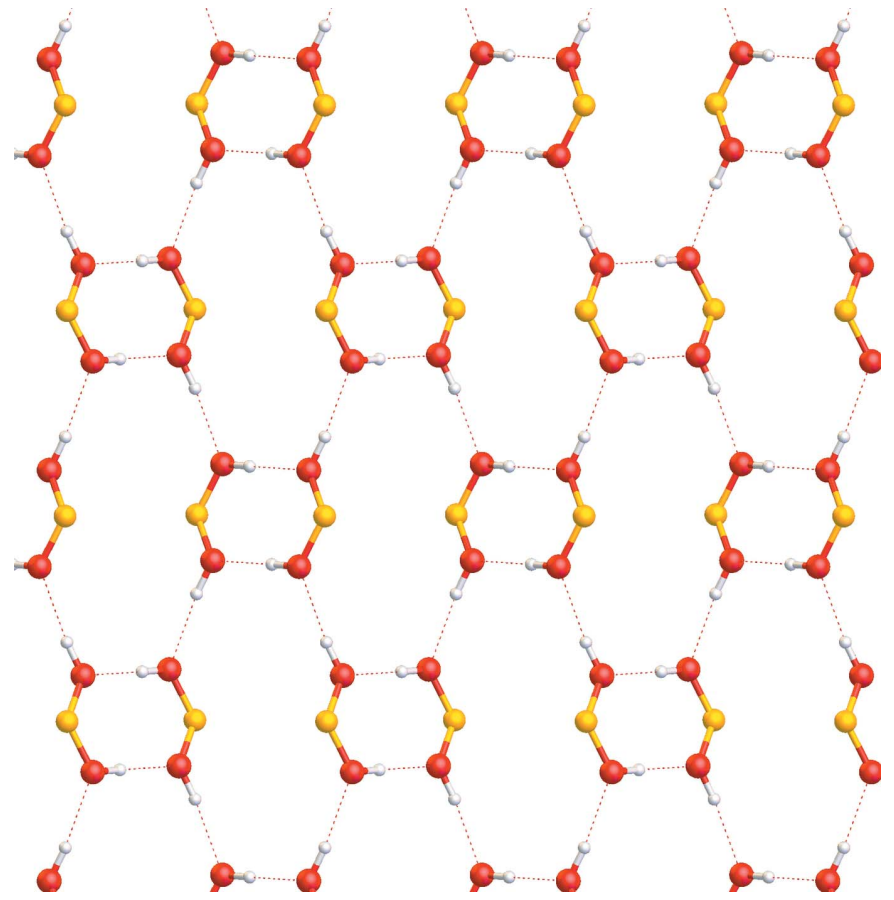

Figure 6

Layered network of hydrogen bonds in the monoclinic form. View is along the [001] axis, only boronic acid groups are shown.
Table 1

Hydrogen-bond geometry $\left(\AA,^{\circ}\right)$ for the orthorhombic polymorph.

\begin{tabular}{lllll}
\hline$D-\mathrm{H} \cdots A$ & $D-\mathrm{H}$ & $\mathrm{H} \cdots A$ & $D \cdots A$ & $D-\mathrm{H} \cdots A$ \\
\hline $\mathrm{O} 1-\mathrm{H} 1 \cdots \mathrm{O} 2^{\mathrm{i}}$ & $0.81(4)$ & $1.98(4)$ & $2.766(2)$ & $165(4)$ \\
$\mathrm{O} 2-\mathrm{H} 2 \cdots \mathrm{O} 3$ & $0.90(3)$ & $1.86(3)$ & $2.750(3)$ & $171(3)$ \\
$\mathrm{O} 3-\mathrm{H} 3 \cdots \mathrm{O} 4{ }^{\mathrm{ii}}$ & $0.96(4)$ & $1.82(4)$ & $2.761(2)$ & $167(3)$ \\
$\mathrm{O} 4-\mathrm{H} 4 \cdots \mathrm{O} 1$ & $0.89(4)$ & $1.85(4)$ & $2.739(3)$ & $175(3)$ \\
$\mathrm{C} 9-\mathrm{H} 9 \cdots \mathrm{O} 2$ & 0.95 & 2.45 & $3.092(3)$ & 124 \\
$\mathrm{C} 19-\mathrm{H} 19 \cdots \mathrm{O} 4$ & 0.95 & 2.42 & $3.063(3)$ & 125 \\
\hline
\end{tabular}

Symmetry codes: (i) $x+\frac{1}{2},-y+\frac{3}{2}, z$; (ii) $x-\frac{1}{2},-y+\frac{1}{2}, z$.

Table 2

Hydrogen-bond geometry $\left(\AA,^{\circ}\right)$ for the monoclinic polymorph.

\begin{tabular}{lllll}
\hline$D-\mathrm{H} \cdots A$ & $D-\mathrm{H}$ & $\mathrm{H} \cdots A$ & $D \cdots A$ & $D-\mathrm{H} \cdots A$ \\
\hline $\mathrm{O} 1-\mathrm{H} 1 \cdots \mathrm{O} 2^{\mathrm{i}}$ & $0.897(18)$ & $1.846(18)$ & $2.7411(13)$ & $176.3(17)$ \\
$\mathrm{O} 2-\mathrm{H} 2 \cdots \mathrm{O} 1^{\mathrm{ii}}$ & $0.888(19)$ & $1.891(19)$ & $2.7607(11)$ & $166.0(17)$ \\
$\mathrm{C} 9-\mathrm{H} 9 \cdots \mathrm{O} 1$ & $0.98(1)$ & $2.43(1)$ & $3.0911(15)$ & $124(1)$ \\
\hline
\end{tabular}

Symmetry codes: (i) $-x+1,-y,-z+1$; (ii) $x,-y+\frac{1}{2}, z-\frac{1}{2}$.

There are no directional intermolecular interactions between adjacent layers and, therefore, no strong interactions between them. However, these layers are shifted with respect to the [010] axis (compare Figs. 9 and 10), resulting in a slightly

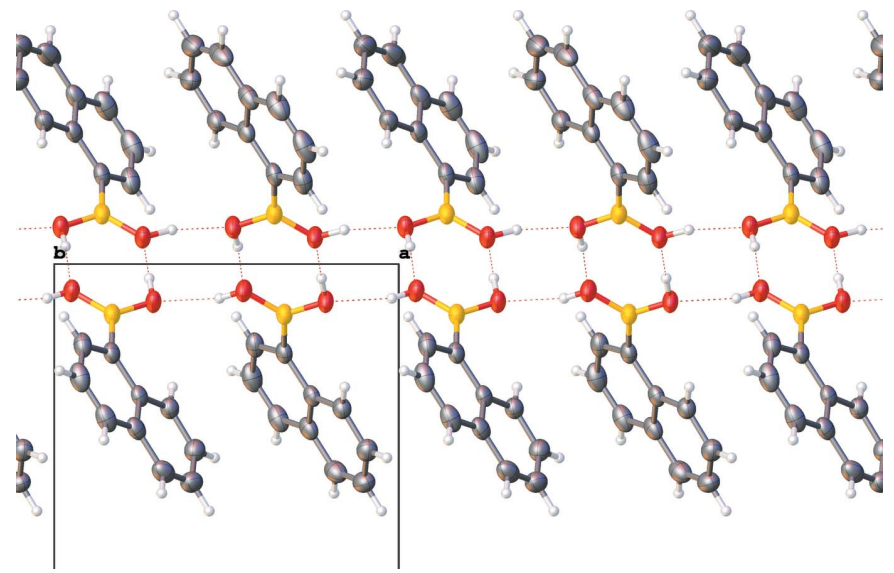

Figure 7

Packing of the orthorhombic form. View is along the [010] axis.

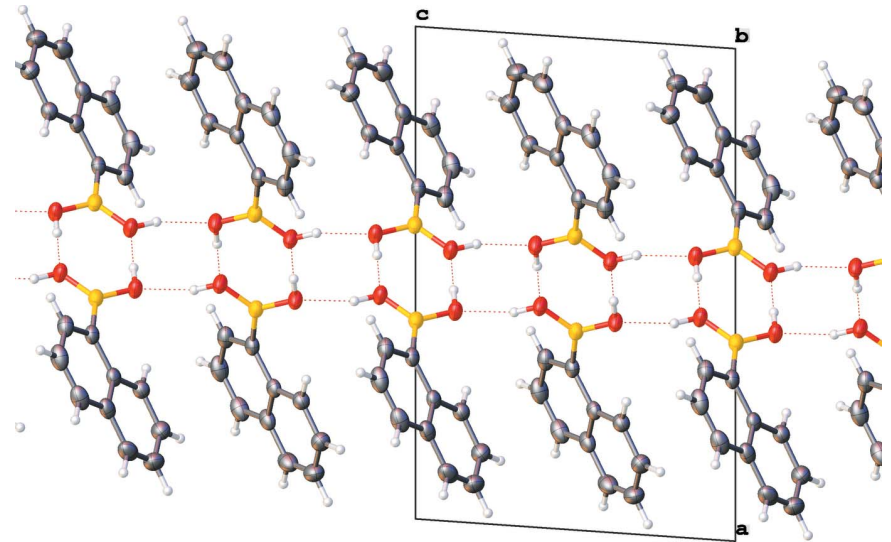

Figure 8

Packing of the monoclinic form. View is along the [010] axis. 
Table 3

Experimental details.

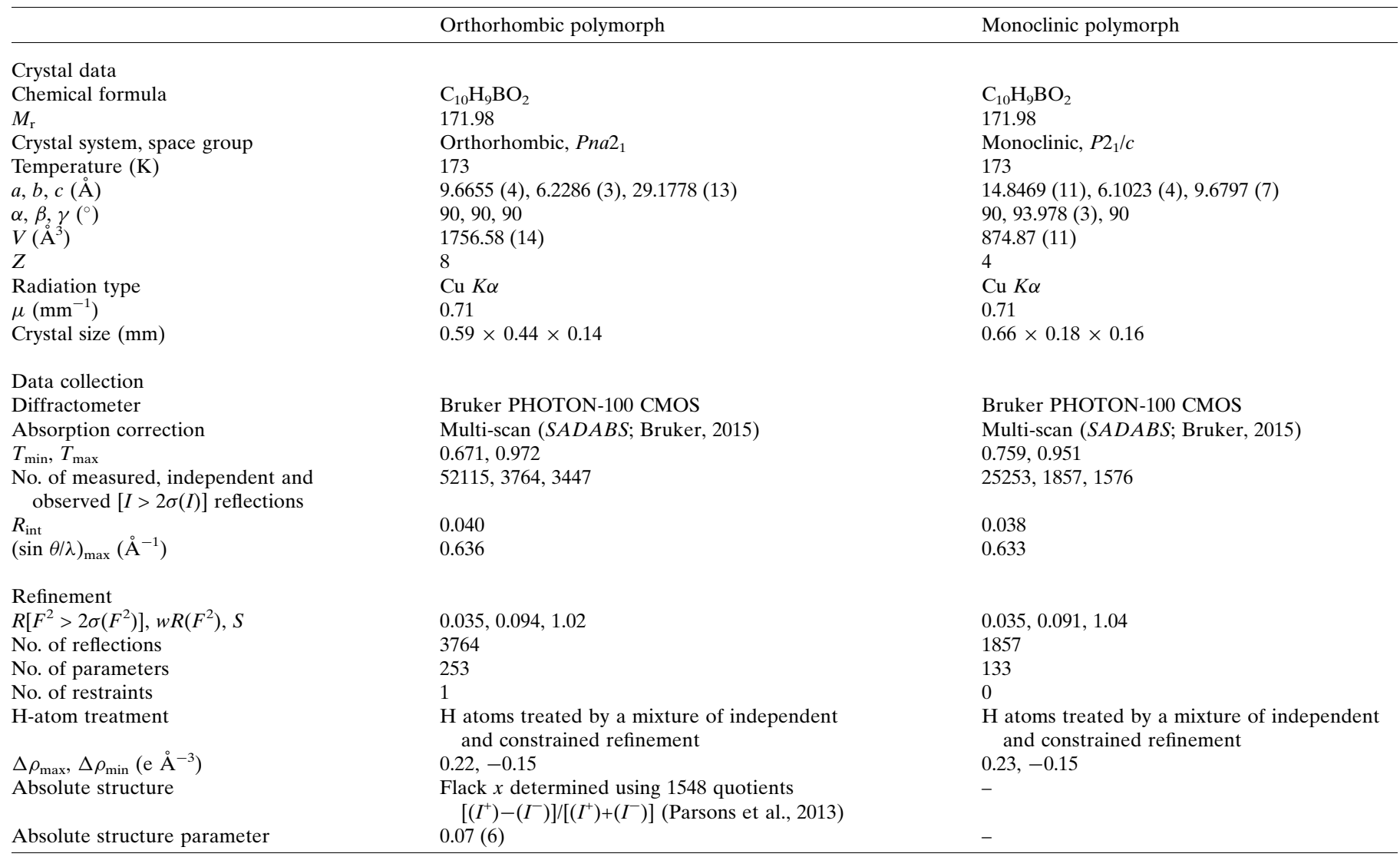

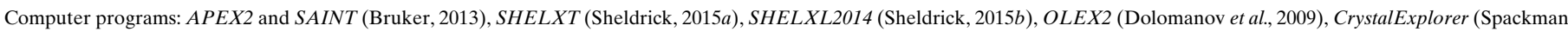
\& Jayatilaka, 2009) and PLATON (Spek, 2009).

more effective packing of the monoclinic structure (packing index $=0.692$ ) (Kitaigorodskii, 1961; Spek, 2009) compared to the orthorhombic structure (packing index $=0.688$ ). This layer-shift is the only visible difference between the two forms.

\section{Database survey}

There are no naphthalene boronic acid structures deposited in the Cambridge Structural Database (CSD Version 5.37; Groom et al., 2016). The simplest arylboronic acid, phenylboronic acid, crystallizes in a non-centrosymmetric ortho-
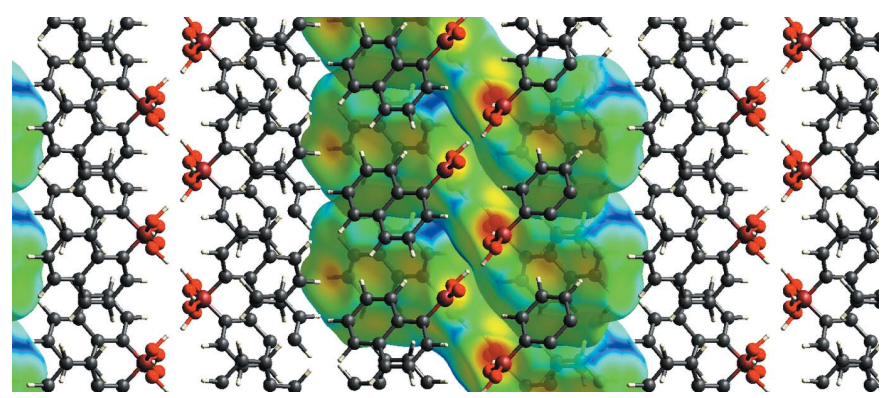

Figure 9

Packing diagram of the orthorhombic form. View is along the [100] axis. Hirshfeld surface shown for some molecules. rhombic space group (refcodes PHBORA and PHBORA01). Instead of a layered network, its molecules form an infinitive chain in the crystal (Cyránski et al., 2008; Rettig \& Trotter, 1977).

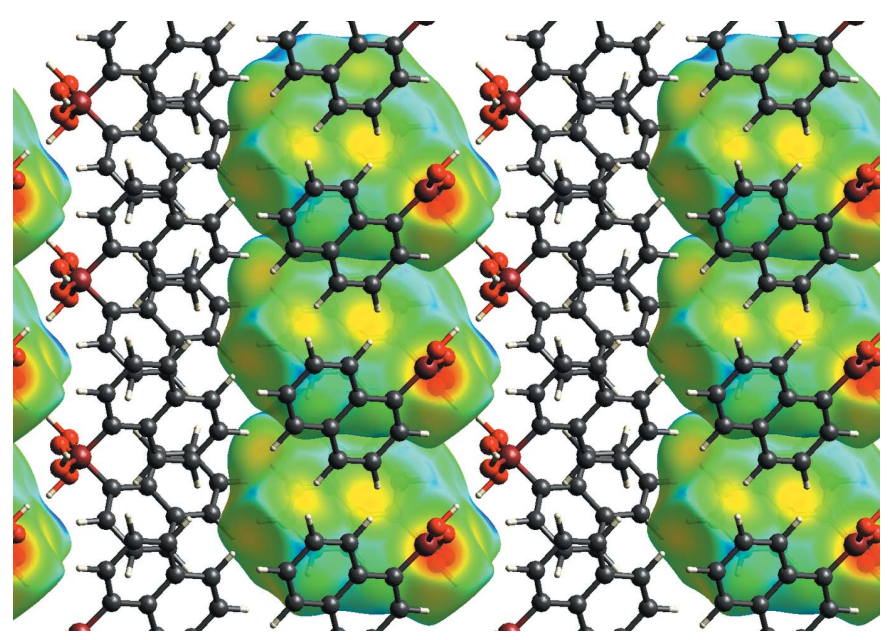

Figure 10

Packing diagram of the monoclinic form. View is along the [001] axis. Hirshfeld surface shown for some molecules. 


\section{Refinement}

Crystal data, data collection and structure refinement details are summarized in Table 3. All hydrogen atoms of hydroxyl groups were refined in an isotropic approximation. Aromatic hydrogen atoms were refined with riding coordinates and $U_{\text {iso }}(\mathrm{H})=1.2 U_{\text {iso }}(\mathrm{C})$.

\section{Acknowledgements}

Financial support from the State University of New York for acquisition and maintenance of the X-ray diffractometer is gratefully acknowledged.

\section{References}

Bruker (2013). APEX2 and SAINT. Bruker AXS Inc., Madison, Wisconsin, USA.

Bruker (2015). SADABS. Bruker AXS Inc., Madison, Wisconsin, USA.
Cyrański, M. K., Jezierska, A., Klimentowska, P., Panek, J. J. \& Sporzyński, A. (2008). J. Phys. Org. Chem. 21, 472-482.

Dolomanov, O. V., Bourhis, L. J., Gildea, R. J., Howard, J. A. K. \& Puschmann, H. (2009). J. Appl. Cryst. 42, 339-341.

Gao, X., Zhang, Y. \& Wang, B. (2003). Org. Lett. 5, 4615-4618.

Groom, C. R., Bruno, I. J., Lightfoot, M. P. \& Ward, S. C. (2016). Acta Cryst. B72, 171-179.

Hall, D. G. (2011). Editor. Boronic Acids: Preparation and Applications in Organic Synthesis, Medicine and Materials. New York: Wiley-VCH.

Kitaigorodskii, A. I. (1961). In Organic Chemical Crystallography. New York: Consultants Bureau.

König, W. \& Scharrnbeck, W. (1930). J. Prakt. Chem. 128, 153-170.

Michaelis, A. (1894). Ber. Dtsch. Chem. Ges. 27, 244-262.

Parsons, S., Flack, H. D. \& Wagner, T. (2013). Acta Cryst. B69, 249259.

Rettig, S. J. \& Trotter, J. (1977). Can. J. Chem. 55, 3071-3075.

Sheldrick, G. M. (2015a). Acta Cryst. A71, 3-8.

Sheldrick, G. M. (2015b). Acta Cryst. C71, 3-8.

Spackman, M. A. \& Jayatilaka, D. (2009). CrystEngComm, 11, 19-32. Spek, A. L. (2009). Acta Cryst. D65, 148-155. 


\section{supporting information}

Acta Cryst. (2016). E72, 1285-1289 [https://doi.org/10.1107/S2056989016012494]

\section{Two forms of (naphthalen-1-yl)boronic acid}

\section{Kayleigh Bemisderfer and Alexander Y. Nazarenko}

\section{Computing details}

For both compounds, data collection: APEX2 (Bruker, 2013); cell refinement: SAINT (Bruker, 2013); data reduction: SAINT (Bruker, 2013); program(s) used to solve structure: SHELXT (Sheldrick, 2015a); program(s) used to refine structure: SHELXL2014 (Sheldrick, 2015b). Molecular graphics: OLEX2 (Dolomanov et al., 2009) and CrystalExplorer (Spackman \& Jayatilaka, 2009) for (1); OLEX2 (Dolomanov et al., 2009) for (2). Software used to prepare material for publication: OLEX2 (Dolomanov et al., 2009) and PLATON (Spek, 2009) for (1); OLEX2 (Dolomanov et al., 2009) for (2).

(1) (Naphthalen-1-yl)boronic acid

Crystal data

$\mathrm{C}_{10} \mathrm{H}_{9} \mathrm{BO}_{2}$

$M_{r}=171.98$

Orthorhombic, Pna $_{1}$

$a=9.6655(4) \AA$

$b=6.2286(3) \AA$

$c=29.1778(13) \AA$

$V=1756.58(14) \AA^{3}$

$Z=8$

$F(000)=720$

\section{Data collection}

Bruker PHOTON-100 CMOS diffractometer

Radiation source: sealedtube $\varphi$ and $\omega$ scans

Absorption correction: multi-scan

(SADABS; Bruker, 2015)

$T_{\text {min }}=0.671, T_{\max }=0.972$

52115 measured reflections

\section{Refinement}

Refinement on $F^{2}$

Least-squares matrix: full

$R\left[F^{2}>2 \sigma\left(F^{2}\right)\right]=0.035$

$w R\left(F^{2}\right)=0.094$

$S=1.02$

3764 reflections

253 parameters

1 restraint

Hydrogen site location: mixed
$D_{\mathrm{x}}=1.301 \mathrm{Mg} \mathrm{m}^{-3}$

$\mathrm{Cu} K \alpha$ radiation, $\lambda=1.54178 \AA$

Cell parameters from 9127 reflections

$\theta=3.0-78.2^{\circ}$

$\mu=0.71 \mathrm{~mm}^{-1}$

$T=173 \mathrm{~K}$

Plate, colourless

$0.59 \times 0.44 \times 0.14 \mathrm{~mm}$

3764 independent reflections

3447 reflections with $I>2 \sigma(I)$

$R_{\text {int }}=0.040$

$\theta_{\max }=78.7^{\circ}, \theta_{\min }=3.0^{\circ}$

$h=-12 \rightarrow 12$

$k=-7 \rightarrow 7$

$l=-36 \rightarrow 36$

$\mathrm{H}$ atoms treated by a mixture of independent

and constrained refinement

$w=1 /\left[\sigma^{2}\left(F_{\mathrm{o}}^{2}\right)+(0.0563 P)^{2}+0.2507 P\right]$

where $P=\left(F_{\mathrm{o}}{ }^{2}+2 F_{\mathrm{c}}{ }^{2}\right) / 3$

$(\Delta / \sigma)_{\max }<0.001$

$\Delta \rho_{\max }=0.22 \mathrm{e} \AA^{-3}$

$\Delta \rho_{\min }=-0.15$ e $\AA^{-3}$ 
Absolute structure: Flack $x$ determined using 1548 quotients $\left[\left(I^{+}\right)-\left(I^{-}\right)\right] /\left[\left(I^{+}\right)+\left(I^{-}\right)\right]$(Parsons et al., 2013)

Absolute structure parameter: 0.07 (6)

\section{Special details}

Geometry. All esds (except the esd in the dihedral angle between two 1.s. planes) are estimated using the full covariance matrix. The cell esds are taken into account individually in the estimation of esds in distances, angles and torsion angles; correlations between esds in cell parameters are only used when they are defined by crystal symmetry. An approximate (isotropic) treatment of cell esds is used for estimating esds involving l.s. planes.

Fractional atomic coordinates and isotropic or equivalent isotropic displacement parameters $\left(\AA^{2}\right)$

\begin{tabular}{|c|c|c|c|c|}
\hline & $x$ & $y$ & $z$ & $U_{\text {iso }} * / U_{\text {eq }}$ \\
\hline $\mathrm{O} 3$ & $0.24283(16)$ & $0.3150(3)$ & $0.47433(6)$ & $0.0415(4)$ \\
\hline $\mathrm{H} 3$ & $0.158(4)$ & $0.244(6)$ & $0.4672(11)$ & $0.062 *$ \\
\hline $\mathrm{O} 4$ & $0.48065(16)$ & $0.3548(3)$ & $0.46451(6)$ & $0.0398(4)$ \\
\hline $\mathrm{H} 4$ & $0.467(4)$ & $0.466(6)$ & $0.4831(12)$ & $0.060^{*}$ \\
\hline $\mathrm{C} 11$ & $0.3612(2)$ & $0.0750(4)$ & $0.41412(9)$ & $0.0367(5)$ \\
\hline $\mathrm{C} 12$ & $0.2783(3)$ & $-0.1033(4)$ & $0.42012(11)$ & $0.0472(6)$ \\
\hline H12 & $0.222(2)$ & $-0.1146(6)$ & $0.4475(10)$ & $0.057^{*}$ \\
\hline $\mathrm{C} 13$ & $0.2731(3)$ & $-0.2707(5)$ & $0.38704(13)$ & $0.0581(8)$ \\
\hline H13 & $0.220(2)$ & $-0.383(5)$ & $0.3918(2)$ & $0.070^{*}$ \\
\hline $\mathrm{C} 14$ & $0.3497(3)$ & $-0.2587(5)$ & $0.34829(12)$ & $0.0549(8)$ \\
\hline H14 & $0.3444(4)$ & $-0.387(4)$ & $0.3237(8)$ & $0.066^{*}$ \\
\hline $\mathrm{C} 15$ & $0.4357(3)$ & $-0.0818(4)$ & $0.33976(10)$ & $0.0451(6)$ \\
\hline $\mathrm{C} 16$ & $0.5152(3)$ & $-0.0637(6)$ & $0.29893(10)$ & $0.0566(8)$ \\
\hline H16 & $0.5105(4)$ & $-0.182(4)$ & $0.2757(9)$ & $0.068^{*}$ \\
\hline $\mathrm{C} 17$ & $0.5970(3)$ & $0.1086(6)$ & $0.29054(10)$ & $0.0555(8)$ \\
\hline H17 & $0.653(2)$ & $0.1169(6)$ & $0.2611(11)$ & $0.067^{*}$ \\
\hline C18 & $0.6038(3)$ & $0.2752(5)$ & $0.32268(9)$ & $0.0485(7)$ \\
\hline H18 & $0.666(2)$ & $0.407(5)$ & $0.3163(2)$ & $0.058^{*}$ \\
\hline $\mathrm{C} 19$ & $0.5287(2)$ & $0.2649(4)$ & $0.36254(8)$ & $0.0389(5)$ \\
\hline H19 & 0.5350 & 0.3799 & 0.3838 & $0.047^{*}$ \\
\hline $\mathrm{C} 20$ & $0.4422(2)$ & $0.0882(4)$ & $0.37291(8)$ & $0.0358(5)$ \\
\hline B2 & $0.3611(3)$ & $0.2548(5)$ & $0.45182(10)$ & $0.0352(6)$ \\
\hline $\mathrm{O} 1$ & $0.44946(17)$ & $0.6845(3)$ & $0.52575(6)$ & $0.0410(4)$ \\
\hline H1 & $0.520(4)$ & $0.747(6)$ & $0.5329(13)$ & $0.061^{*}$ \\
\hline $\mathrm{O} 2$ & $0.21178(16)$ & $0.6447(3)$ & $0.53618(6)$ & $0.0393(4)$ \\
\hline $\mathrm{H} 2$ & $0.224(4)$ & $0.548(5)$ & $0.5138(12)$ & $0.059^{*}$ \\
\hline $\mathrm{C} 1$ & $0.3326(2)$ & $0.9282(4)$ & $0.58539(8)$ & $0.0337(5)$ \\
\hline $\mathrm{C} 2$ & $0.4169(2)$ & $1.1032(4)$ & $0.57782(10)$ & $0.0417(6)$ \\
\hline $\mathrm{H} 2 \mathrm{~A}$ & 0.4712 & 1.1076 & 0.5507 & $0.050^{*}$ \\
\hline $\mathrm{C} 3$ & $0.4253(3)$ & $1.2758(4)$ & $0.60894(11)$ & $0.0484(7)$ \\
\hline $\mathrm{H} 3 \mathrm{~A}$ & 0.4842 & 1.3941 & 0.6025 & $0.058^{*}$ \\
\hline $\mathrm{C} 4$ & $0.3491(3)$ & $1.2734(4)$ & $0.64824(11)$ & $0.0460(6)$ \\
\hline $\mathrm{H} 4 \mathrm{~A}$ & 0.3552 & 1.3901 & 0.6691 & $0.055^{*}$ \\
\hline $\mathrm{C} 5$ & $0.2613(2)$ & $1.0981(4)$ & $0.65810(9)$ & $0.0386(5)$ \\
\hline C6 & $0.1826(3)$ & $1.0920(5)$ & $0.69941(9)$ & $0.0462(6)$ \\
\hline
\end{tabular}


supporting information

$\begin{array}{lllll}\text { H6 } & 0.1876 & 1.2092 & 0.7202 & 0.055^{*} \\ \text { C7 } & 0.1010(3) & 0.9220(5) & 0.70950(9) & 0.0482(6) \\ \text { H7 } & 0.0499 & 0.9201 & 0.7373 & 0.058^{*} \\ \text { C8 } & 0.0916(3) & 0.7484(5) & 0.67893(9) & 0.0437(6) \\ \text { H8 } & 0.0343 & 0.6294 & 0.6862 & 0.052^{*} \\ \text { C9 } & 0.1647(2) & 0.7492(4) & 0.63858(8) & 0.0361(5) \\ \text { H9 } & 0.1567 & 0.6308 & 0.6182 & 0.043^{*} \\ \text { C10 } & 0.2520(2) & 0.9235(4) & 0.62674(8) & 0.0328(5) \\ \text { B1 } & 0.3317(3) & 0.7451(5) & 0.54832(9) & 0.0347(5)\end{array}$

Atomic displacement parameters $\left(\AA^{2}\right)$

\begin{tabular}{lllllll}
\hline & $U^{11}$ & $U^{22}$ & $U^{33}$ & $U^{12}$ & $U^{13}$ & $U^{23}$ \\
\hline O3 & $0.0220(7)$ & $0.0608(11)$ & $0.0418(9)$ & $-0.0033(8)$ & $0.0009(6)$ & $-0.0085(8)$ \\
O4 & $0.0227(7)$ & $0.0537(10)$ & $0.0430(10)$ & $-0.0004(8)$ & $-0.0010(6)$ & $-0.0104(8)$ \\
C11 & $0.0239(10)$ & $0.0383(12)$ & $0.0479(13)$ & $0.0028(9)$ & $-0.0073(9)$ & $-0.0001(11)$ \\
C12 & $0.0329(11)$ & $0.0453(14)$ & $0.0633(17)$ & $-0.0010(11)$ & $-0.0080(12)$ & $0.0086(12)$ \\
C13 & $0.0457(15)$ & $0.0360(13)$ & $0.093(3)$ & $-0.0063(12)$ & $-0.0258(16)$ & $0.0035(14)$ \\
C14 & $0.0487(16)$ & $0.0426(14)$ & $0.073(2)$ & $0.0077(12)$ & $-0.0200(14)$ & $-0.0160(14)$ \\
C15 & $0.0370(12)$ & $0.0442(14)$ & $0.0541(16)$ & $0.0115(10)$ & $-0.0158(11)$ & $-0.0128(12)$ \\
C16 & $0.0511(16)$ & $0.0719(19)$ & $0.0469(16)$ & $0.0244(15)$ & $-0.0136(12)$ & $-0.0248(15)$ \\
C17 & $0.0450(15)$ & $0.079(2)$ & $0.0421(15)$ & $0.0145(15)$ & $-0.0012(11)$ & $-0.0093(14)$ \\
C18 & $0.0381(14)$ & $0.0660(18)$ & $0.0414(14)$ & $0.0042(13)$ & $-0.0002(10)$ & $-0.0029(12)$ \\
C19 & $0.0309(12)$ & $0.0461(13)$ & $0.0396(12)$ & $0.0038(10)$ & $-0.0037(10)$ & $-0.0061(10)$ \\
C20 & $0.0270(9)$ & $0.0387(12)$ & $0.0417(13)$ & $0.0079(9)$ & $-0.0074(9)$ & $-0.0064(10)$ \\
B2 & $0.0231(12)$ & $0.0454(14)$ & $0.0371(13)$ & $0.0006(11)$ & $-0.0011(10)$ & $0.0038(11)$ \\
O1 & $0.0234(7)$ & $0.0568(11)$ & $0.0427(10)$ & $-0.0046(8)$ & $0.0025(6)$ & $-0.0069(8)$ \\
O2 & $0.0212(7)$ & $0.0542(10)$ & $0.0424(9)$ & $0.0002(7)$ & $0.0011(6)$ & $-0.0093(8)$ \\
C1 & $0.0242(9)$ & $0.0371(11)$ & $0.0399(12)$ & $0.0030(9)$ & $-0.0045(8)$ & $0.0034(10)$ \\
C2 & $0.0284(11)$ & $0.0433(13)$ & $0.0533(15)$ & $-0.0009(10)$ & $-0.0037(10)$ & $0.0086(11)$ \\
C3 & $0.0369(14)$ & $0.0342(12)$ & $0.0741(19)$ & $-0.0046(11)$ & $-0.0109(13)$ & $0.0058(12)$ \\
C4 & $0.0382(14)$ & $0.0351(12)$ & $0.0647(18)$ & $0.0031(11)$ & $-0.0148(11)$ & $-0.0056(12)$ \\
C5 & $0.0313(11)$ & $0.0391(12)$ & $0.0453(14)$ & $0.0080(10)$ & $-0.0107(9)$ & $-0.0030(10)$ \\
C6 & $0.0454(14)$ & $0.0501(14)$ & $0.0430(14)$ & $0.0113(12)$ & $-0.0084(11)$ & $-0.0114(11)$ \\
C7 & $0.0440(14)$ & $0.0630(16)$ & $0.0377(13)$ & $0.0078(13)$ & $0.0009(10)$ & $-0.0025(12)$ \\
C8 & $0.0379(13)$ & $0.0500(14)$ & $0.0433(13)$ & $0.0023(12)$ & $0.0010(10)$ & $0.0045(10)$ \\
C9 & $0.0303(11)$ & $0.0386(11)$ & $0.0395(12)$ & $0.0016(9)$ & $-0.0025(9)$ & $-0.0011(9)$ \\
C10 & $0.0248(9)$ & $0.0345(11)$ & $0.0392(12)$ & $0.0050(9)$ & $-0.0070(8)$ & $0.0009(9)$ \\
B1 & $0.0228(12)$ & $0.0439(13)$ & $0.0374(13)$ & $0.0014(10)$ & $-0.0004(9)$ & $0.0007(11)$ \\
& & & & & &
\end{tabular}

Geometric parameters $\left(\AA,{ }^{o}\right)$

\begin{tabular}{llll}
\hline $\mathrm{O} 3-\mathrm{H} 3$ & $0.95(4)$ & $\mathrm{O} 1-\mathrm{H} 1$ & $0.81(4)$ \\
$\mathrm{O} 3-\mathrm{B} 2$ & $1.371(3)$ & $\mathrm{O} 1-\mathrm{B} 1$ & $1.368(3)$ \\
$\mathrm{O} 4-\mathrm{H} 4$ & $0.89(4)$ & $\mathrm{O} 2-\mathrm{H} 2$ & $0.90(3)$ \\
$\mathrm{O} 4-\mathrm{B} 2$ & $1.363(3)$ & $\mathrm{O} 2-\mathrm{B} 1$ & $1.364(3)$ \\
$\mathrm{C} 11-\mathrm{C} 12$ & $1.381(4)$ & $\mathrm{C} 1-\mathrm{C} 2$ & $1.379(4)$ \\
$\mathrm{C} 11-\mathrm{C} 20$ & $1.438(3)$ & $\mathrm{C} 1-\mathrm{C} 10$ & $1.436(3)$
\end{tabular}




\begin{tabular}{|c|c|c|c|}
\hline $\mathrm{C} 11-\mathrm{B} 2$ & $1.570(4)$ & $\mathrm{C} 1-\mathrm{B} 1$ & $1.572(4)$ \\
\hline $\mathrm{C} 12-\mathrm{H} 12$ & $0.97(3)$ & $\mathrm{C} 2-\mathrm{H} 2 \mathrm{~A}$ & 0.9500 \\
\hline $\mathrm{C} 12-\mathrm{C} 13$ & $1.422(5)$ & $\mathrm{C} 2-\mathrm{C} 3$ & $1.410(4)$ \\
\hline C13-H13 & $0.88(4)$ & $\mathrm{C} 3-\mathrm{H} 3 \mathrm{~A}$ & 0.9500 \\
\hline $\mathrm{C} 13-\mathrm{C} 14$ & $1.353(5)$ & $\mathrm{C} 3-\mathrm{C} 4$ & $1.363(4)$ \\
\hline C14-H14 & $1.07(4)$ & $\mathrm{C} 4-\mathrm{H} 4 \mathrm{~A}$ & 0.9500 \\
\hline $\mathrm{C} 14-\mathrm{C} 15$ & $1.403(4)$ & $\mathrm{C} 4-\mathrm{C} 5$ & $1.413(4)$ \\
\hline $\mathrm{C} 15-\mathrm{C} 16$ & $1.422(4)$ & $\mathrm{C} 5-\mathrm{C} 6$ & $1.426(4)$ \\
\hline $\mathrm{C} 15-\mathrm{C} 20$ & $1.435(3)$ & $\mathrm{C} 5-\mathrm{C} 10$ & $1.424(3)$ \\
\hline $\mathrm{C} 16-\mathrm{H} 16$ & $1.00(4)$ & $\mathrm{C} 6-\mathrm{H} 6$ & 0.9500 \\
\hline $\mathrm{C} 16-\mathrm{C} 17$ & $1.355(5)$ & $\mathrm{C} 6-\mathrm{C} 7$ & $1.353(4)$ \\
\hline C17-H17 & $1.02(4)$ & $\mathrm{C} 7-\mathrm{H} 7$ & 0.9500 \\
\hline $\mathrm{C} 17-\mathrm{C} 18$ & $1.400(4)$ & $\mathrm{C} 7-\mathrm{C} 8$ & $1.405(4)$ \\
\hline C18-H18 & $1.03(4)$ & $\mathrm{C} 8-\mathrm{H} 8$ & 0.9500 \\
\hline $\mathrm{C} 18-\mathrm{C} 19$ & $1.373(3)$ & $\mathrm{C} 8-\mathrm{C} 9$ & $1.373(4)$ \\
\hline C19-H19 & 0.9500 & C9- $\mathrm{H} 9$ & 0.9500 \\
\hline $\mathrm{C} 19-\mathrm{C} 20$ & $1.415(3)$ & $\mathrm{C} 9-\mathrm{C} 10$ & $1.417(3)$ \\
\hline $\mathrm{B} 2-\mathrm{O} 3-\mathrm{H} 3$ & $119(2)$ & $\mathrm{B} 1-\mathrm{O} 1-\mathrm{H} 1$ & $116(3)$ \\
\hline $\mathrm{B} 2-\mathrm{O} 4-\mathrm{H} 4$ & $113(2)$ & $\mathrm{B} 1-\mathrm{O} 2-\mathrm{H} 2$ & $113(2)$ \\
\hline $\mathrm{C} 12-\mathrm{C} 11-\mathrm{C} 20$ & $117.9(2)$ & $\mathrm{C} 2-\mathrm{C} 1-\mathrm{C} 10$ & $118.1(2)$ \\
\hline $\mathrm{C} 12-\mathrm{C} 11-\mathrm{B} 2$ & $119.0(2)$ & $\mathrm{C} 2-\mathrm{C} 1-\mathrm{B} 1$ & $117.8(2)$ \\
\hline $\mathrm{C} 20-\mathrm{C} 11-\mathrm{B} 2$ & $123.1(2)$ & $\mathrm{C} 10-\mathrm{C} 1-\mathrm{B} 1$ & $124.1(2)$ \\
\hline $\mathrm{C} 11-\mathrm{C} 12-\mathrm{H} 12$ & 119.2 & $\mathrm{C} 1-\mathrm{C} 2-\mathrm{H} 2 \mathrm{~A}$ & 118.9 \\
\hline $\mathrm{C} 11-\mathrm{C} 12-\mathrm{C} 13$ & $121.6(3)$ & $\mathrm{C} 1-\mathrm{C} 2-\mathrm{C} 3$ & $122.3(3)$ \\
\hline $\mathrm{C} 13-\mathrm{C} 12-\mathrm{H} 12$ & 119.2 & $\mathrm{C} 3-\mathrm{C} 2-\mathrm{H} 2 \mathrm{~A}$ & 118.9 \\
\hline $\mathrm{C} 12-\mathrm{C} 13-\mathrm{H} 13$ & 119.7 & $\mathrm{C} 2-\mathrm{C} 3-\mathrm{H} 3 \mathrm{~A}$ & 119.9 \\
\hline $\mathrm{C} 14-\mathrm{C} 13-\mathrm{C} 12$ & $120.5(3)$ & $\mathrm{C} 4-\mathrm{C} 3-\mathrm{C} 2$ & $120.1(2)$ \\
\hline $\mathrm{C} 14-\mathrm{C} 13-\mathrm{H} 13$ & 119.7 & $\mathrm{C} 4-\mathrm{C} 3-\mathrm{H} 3 \mathrm{~A}$ & 119.9 \\
\hline $\mathrm{C} 13-\mathrm{C} 14-\mathrm{H} 14$ & 119.5 & $\mathrm{C} 3-\mathrm{C} 4-\mathrm{H} 4 \mathrm{~A}$ & 119.9 \\
\hline $\mathrm{C} 13-\mathrm{C} 14-\mathrm{C} 15$ & $121.0(3)$ & $\mathrm{C} 3-\mathrm{C} 4-\mathrm{C} 5$ & $120.3(3)$ \\
\hline $\mathrm{C} 15-\mathrm{C} 14-\mathrm{H} 14$ & 119.5 & $\mathrm{C} 5-\mathrm{C} 4-\mathrm{H} 4 \mathrm{~A}$ & 119.9 \\
\hline $\mathrm{C} 14-\mathrm{C} 15-\mathrm{C} 16$ & $122.1(3)$ & $\mathrm{C} 4-\mathrm{C} 5-\mathrm{C} 6$ & $120.9(2)$ \\
\hline $\mathrm{C} 14-\mathrm{C} 15-\mathrm{C} 20$ & $119.1(3)$ & $\mathrm{C} 4-\mathrm{C} 5-\mathrm{C} 10$ & $119.8(2)$ \\
\hline $\mathrm{C} 16-\mathrm{C} 15-\mathrm{C} 20$ & $118.8(3)$ & $\mathrm{C} 10-\mathrm{C} 5-\mathrm{C} 6$ & $119.3(2)$ \\
\hline $\mathrm{C} 15-\mathrm{C} 16-\mathrm{H} 16$ & 119.0 & $\mathrm{C} 5-\mathrm{C} 6-\mathrm{H} 6$ & 119.5 \\
\hline $\mathrm{C} 17-\mathrm{C} 16-\mathrm{C} 15$ & $122.0(3)$ & $\mathrm{C} 7-\mathrm{C} 6-\mathrm{C} 5$ & $121.1(2)$ \\
\hline $\mathrm{C} 17-\mathrm{C} 16-\mathrm{H} 16$ & 119.0 & $\mathrm{C} 7-\mathrm{C} 6-\mathrm{H} 6$ & 119.5 \\
\hline $\mathrm{C} 16-\mathrm{C} 17-\mathrm{H} 17$ & 120.2 & $\mathrm{C} 6-\mathrm{C} 7-\mathrm{H} 7$ & 119.9 \\
\hline $\mathrm{C} 16-\mathrm{C} 17-\mathrm{C} 18$ & $119.6(3)$ & $\mathrm{C} 6-\mathrm{C} 7-\mathrm{C} 8$ & $120.1(3)$ \\
\hline $\mathrm{C} 18-\mathrm{C} 17-\mathrm{H} 17$ & 120.2 & $\mathrm{C} 8-\mathrm{C} 7-\mathrm{H} 7$ & 119.9 \\
\hline $\mathrm{C} 17-\mathrm{C} 18-\mathrm{H} 18$ & 119.7 & $\mathrm{C} 7-\mathrm{C} 8-\mathrm{H} 8$ & 119.7 \\
\hline $\mathrm{C} 19-\mathrm{C} 18-\mathrm{C} 17$ & $120.5(3)$ & $\mathrm{C} 9-\mathrm{C} 8-\mathrm{C} 7$ & $120.6(3)$ \\
\hline $\mathrm{C} 19-\mathrm{C} 18-\mathrm{H} 18$ & 119.7 & $\mathrm{C} 9-\mathrm{C} 8-\mathrm{H} 8$ & 119.7 \\
\hline $\mathrm{C} 18-\mathrm{C} 19-\mathrm{H} 19$ & 119.0 & $\mathrm{C} 8-\mathrm{C} 9-\mathrm{H} 9$ & 119.4 \\
\hline $\mathrm{C} 18-\mathrm{C} 19-\mathrm{C} 20$ & $122.0(2)$ & $\mathrm{C} 8-\mathrm{C} 9-\mathrm{C} 10$ & $121.2(2)$ \\
\hline $\mathrm{C} 20-\mathrm{C} 19-\mathrm{H} 19$ & 119.0 & $\mathrm{C} 10-\mathrm{C} 9-\mathrm{H} 9$ & 119.4 \\
\hline $\mathrm{C} 15-\mathrm{C} 20-\mathrm{C} 11$ & $119.8(2)$ & $\mathrm{C} 5-\mathrm{C} 10-\mathrm{C} 1$ & $119.3(2)$ \\
\hline
\end{tabular}




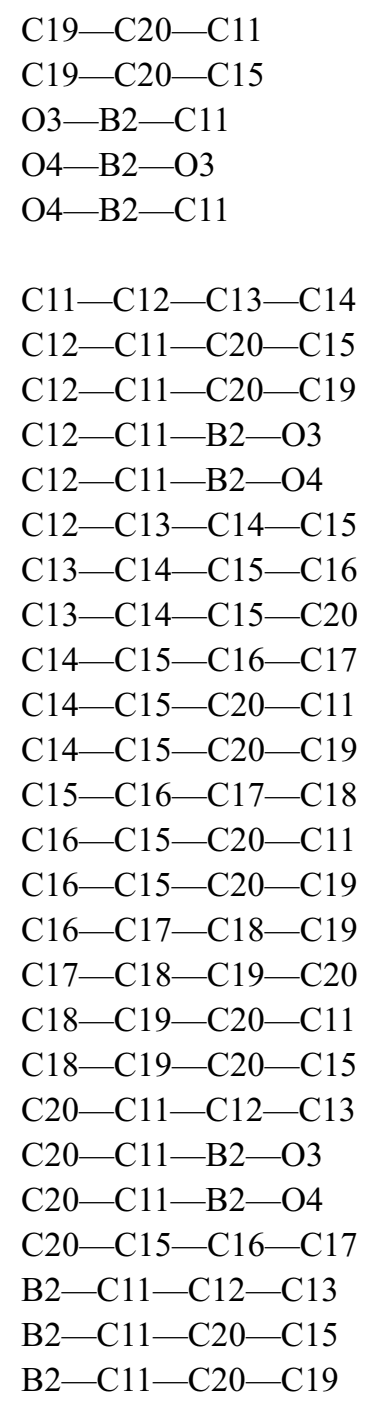

$123.0(2)$

$117.1(2)$

$122.1(2)$

116.9 (2)

$121.1(2)$

$-0.4(4)$

$-0.3(3)$

$178.6(2)$

$-38.2(3)$

$140.1(2)$

$0.0(4)$

$-178.8(3)$

0.2 (4)

$179.2(3)$

0.0 (3)

$-179.1(2)$

$-0.3(4)$

$179.0(2)$

0.0 (3)

$0.2(4)$

0.0 (4)

$-179.1(2)$

$-0.1(3)$

0.5 (3)

140.8 (2)

$-41.0(3)$

$0.2(4)$

$179.6(2)$

$-179.3(2)$

$-0.3(3)$

$$
\begin{aligned}
& \mathrm{C} 9-\mathrm{C} 10-\mathrm{C} 1 \\
& \mathrm{C} 9-\mathrm{C} 10-\mathrm{C} 5 \\
& \mathrm{O} 1-\mathrm{B} 1-\mathrm{C} 1 \\
& \mathrm{O} 2-\mathrm{B} 1-\mathrm{O} 1 \\
& \mathrm{O} 2-\mathrm{B} 1-\mathrm{C} 1
\end{aligned}
$$

$$
\mathrm{C} 1-\mathrm{C} 2-\mathrm{C} 3-\mathrm{C} 4
$$$$
\mathrm{C} 2-\mathrm{C} 1-\mathrm{C} 10-\mathrm{C} 5
$$$$
\mathrm{C} 2-\mathrm{C} 1-\mathrm{C} 10-\mathrm{C} 9
$$$$
\mathrm{C} 2-\mathrm{C} 1-\mathrm{B} 1-\mathrm{O} 1
$$$$
\mathrm{C} 2-\mathrm{C} 1-\mathrm{B} 1-\mathrm{O} 2
$$$$
\mathrm{C} 2-\mathrm{C} 3-\mathrm{C} 4-\mathrm{C} 5
$$$$
\mathrm{C} 3-\mathrm{C} 4-\mathrm{C} 5-\mathrm{C} 6
$$$$
\mathrm{C} 3-\mathrm{C} 4-\mathrm{C} 5-\mathrm{C} 10
$$$$
\mathrm{C} 4-\mathrm{C} 5-\mathrm{C} 6-\mathrm{C} 7
$$$$
\mathrm{C} 4-\mathrm{C} 5-\mathrm{C} 10-\mathrm{C} 1
$$$$
\mathrm{C} 4-\mathrm{C} 5-\mathrm{C} 10-\mathrm{C} 9
$$$$
\mathrm{C} 5-\mathrm{C} 6-\mathrm{C} 7-\mathrm{C} 8
$$$$
\mathrm{C} 6-\mathrm{C} 5-\mathrm{C} 10-\mathrm{C} 1
$$$$
\text { C6- } 5-\mathrm{C} 10-\mathrm{C} 9
$$$$
\text { C6-C7-C8-C9 }
$$$$
\mathrm{C} 7-\mathrm{C} 8-\mathrm{C} 9-\mathrm{C} 10
$$$$
\mathrm{C} 8-\mathrm{C} 9-\mathrm{C} 10-\mathrm{C} 1
$$$$
\mathrm{C} 8-\mathrm{C} 9-\mathrm{C} 10-\mathrm{C} 5
$$$$
\mathrm{C} 10-\mathrm{C} 1-\mathrm{C} 2-\mathrm{C} 3
$$$$
\mathrm{C} 10-\mathrm{C} 1-\mathrm{B} 1-\mathrm{O} 1
$$$$
\mathrm{C} 10-\mathrm{C} 1-\mathrm{B} 1-\mathrm{O} 2
$$$$
\mathrm{C} 10-\mathrm{C} 5-\mathrm{C} 6-\mathrm{C} 7
$$$$
\mathrm{B} 1-\mathrm{C} 1-\mathrm{C} 2-\mathrm{C} 3
$$$$
\mathrm{B} 1-\mathrm{C} 1-\mathrm{C} 10-\mathrm{C} 5
$$$$
\mathrm{B} 1-\mathrm{C} 1-\mathrm{C} 10-\mathrm{C} 9
$$

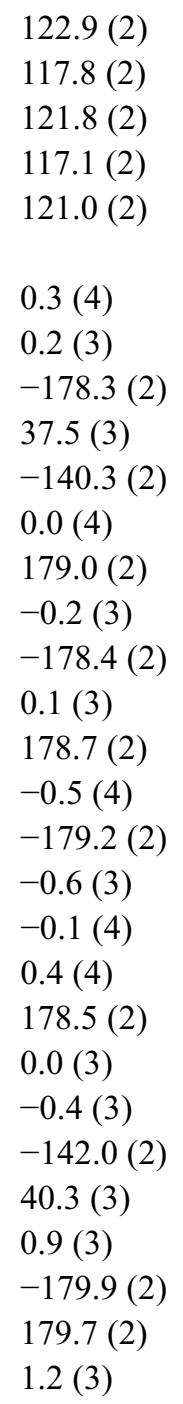

Hydrogen-bond geometry $\left(A,{ }^{\circ}\right)$

\begin{tabular}{lllll}
\hline$D-\mathrm{H} \cdots A$ & $D-\mathrm{H}$ & $\mathrm{H} \cdots A$ & $D \cdots A$ & $D-\mathrm{H} \cdots A$ \\
\hline $\mathrm{O} 1-\mathrm{H} 1 \cdots \mathrm{O} 2^{\mathrm{i}}$ & $0.81(4)$ & $1.98(4)$ & $2.766(2)$ & $165(4)$ \\
$\mathrm{O} 2-\mathrm{H} 2 \cdots \mathrm{O} 3$ & $0.90(3)$ & $1.86(3)$ & $2.750(3)$ & $171(3)$ \\
$\mathrm{O} 3-\mathrm{H} 3 \cdots \mathrm{O} 44^{\mathrm{ii}}$ & $0.96(4)$ & $1.82(4)$ & $2.761(2)$ & $167(3)$ \\
$\mathrm{O} 4-\mathrm{H} 4 \cdots \mathrm{O} 1$ & $0.89(4)$ & $1.85(4)$ & $2.739(3)$ & $175(3)$ \\
$\mathrm{C} 9-\mathrm{H} 9 \cdots \mathrm{O} 2$ & 0.95 & 2.45 & $3.092(3)$ & 124 \\
$\mathrm{C} 19-\mathrm{H} 19 \cdots \mathrm{O} 4$ & 0.95 & 2.42 & $3.063(3)$ & 125 \\
\hline
\end{tabular}

Symmetry codes: (i) $x+1 / 2,-y+3 / 2, z$; (ii) $x-1 / 2,-y+1 / 2, z$. 
(2) (Naphthalen-1-yl)boronic acid

Crystal data

$\mathrm{C}_{10} \mathrm{H}_{9} \mathrm{BO}_{2}$

$M_{r}=171.98$

Monoclinic, $P 2_{1} / c$

$a=14.8469$ (11) $\AA$

$b=6.1023(4) \AA$

$c=9.6797(7) \AA$

$\beta=93.978(3)^{\circ}$

$V=874.87(11) \AA^{3}$

$Z=4$

$F(000)=360$

$D_{\mathrm{x}}=1.306 \mathrm{Mg} \mathrm{m}^{-3}$

$\mathrm{Cu} K \alpha$ radiation, $\lambda=1.54178 \AA$

Cell parameters from 9898 reflections

$\theta=3.0-77.0^{\circ}$

$\mu=0.71 \mathrm{~mm}^{-1}$

$T=173 \mathrm{~K}$

Prism, colourless

$0.66 \times 0.18 \times 0.16 \mathrm{~mm}$

Data collection

Bruker PHOTON-100 CMOS

diffractometer

Radiation source: sealedtube

$\varphi$ and $\omega$ scans

Absorption correction: multi-scan

(SADABS; Bruker, 2015)

$T_{\min }=0.759, T_{\max }=0.951$

25253 measured reflections

1857 independent reflections

1576 reflections with $I>2 \sigma(I)$

$R_{\text {int }}=0.038$

$\theta_{\max }=77.4^{\circ}, \theta_{\min }=3.0^{\circ}$

$h=-18 \rightarrow 18$

$k=-7 \rightarrow 7$

$l=-12 \rightarrow 11$

\section{Refinement}

Refinement on $F^{2}$

Least-squares matrix: full

$R\left[F^{2}>2 \sigma\left(F^{2}\right)\right]=0.035$

$w R\left(F^{2}\right)=0.091$

$S=1.04$

1857 reflections

133 parameters

0 restraints

Hydrogen site location: mixed

$\mathrm{H}$ atoms treated by a mixture of independent and constrained refinement

$w=1 /\left[\sigma^{2}\left(F_{\mathrm{o}}^{2}\right)+(0.0435 P)^{2}+0.2015 P\right]$

where $P=\left(F_{\mathrm{o}}{ }^{2}+2 F_{\mathrm{c}}{ }^{2}\right) / 3$

$(\Delta / \sigma)_{\max }<0.001$

$\Delta \rho_{\max }=0.23 \mathrm{e} \AA^{-3}$

$\Delta \rho_{\min }=-0.15$ e $\AA^{-3}$

Special details

Geometry. All esds (except the esd in the dihedral angle between two 1.s. planes) are estimated using the full covariance matrix. The cell esds are taken into account individually in the estimation of esds in distances, angles and torsion angles; correlations between esds in cell parameters are only used when they are defined by crystal symmetry. An approximate (isotropic) treatment of cell esds is used for estimating esds involving l.s. planes.

Fractional atomic coordinates and isotropic or equivalent isotropic displacement parameters $\left(\AA^{2}\right)$

\begin{tabular}{lllll}
\hline & $x$ & $y$ & $z$ & $U_{\text {iso }} * / U_{\text {eq }}$ \\
\hline O1 & $0.42757(6)$ & $0.14734(15)$ & $0.62491(7)$ & $0.0348(2)$ \\
H1 & $0.4697(12)$ & $0.043(3)$ & $0.6194(17)$ & $0.058(5)^{*}$ \\
O2 & $0.44930(6)$ & $0.18505(15)$ & $0.39004(8)$ & $0.0361(2)$ \\
H2 & $0.4335(12)$ & $0.247(3)$ & $0.309(2)$ & $0.067(5)^{*}$ \\
C1 & $0.32826(8)$ & $0.42799(19)$ & $0.49000(11)$ & $0.0301(3)$ \\
C2 & $0.34171(9)$ & $0.6083(2)$ & $0.40723(12)$ & $0.0374(3)$ \\
H2A & $0.3975(9)$ & $0.6190(3)$ & $0.3608(7)$ & $0.045^{*}$ \\
C3 & $0.27729(10)$ & $0.7770(2)$ & $0.38806(13)$ & $0.0441(3)$ \\
H3 & $0.2893(2)$ & $0.903(2)$ & $0.3287(10)$ & $0.053^{*}$ \\
C4 & $0.19841(10)$ & $0.7660(2)$ & $0.45170(13)$ & $0.0431(3)$
\end{tabular}


supporting information

$\begin{array}{lllll}\text { H4 } & 0.1527(8) & 0.887(2) & 0.4375(3) & 0.052^{*} \\ \text { C5 } & 0.18023(8) & 0.5861(2) & 0.53761(12) & 0.0356(3) \\ \text { C6 } & 0.09731(9) & 0.5709(2) & 0.60194(14) & 0.0445(3) \\ \text { H6 } & 0.0525(8) & 0.688(2) & 0.5877(3) & 0.053^{*} \\ \text { C7 } & 0.07934(9) & 0.3962(3) & 0.68300(14) & 0.0477(3) \\ \text { H7 } & 0.0219(10) & 0.3879(3) & 0.7264(8) & 0.057^{*} \\ \text { C8 } & 0.14332(9) & 0.2281(2) & 0.70431(13) & 0.0411(3) \\ \text { H8 } & 0.1299(2) & 0.100(2) & 0.7651(10) & 0.049^{*} \\ \text { C9 } & 0.22382(8) & 0.2367(2) & 0.64330(11) & 0.0327(3) \\ \text { H9 } & 0.2672(6) & 0.1175(18) & 0.6589(3) & 0.039^{*} \\ \text { C10 } & 0.24527(8) & 0.41549(18) & 0.55791(11) & 0.0296(3) \\ \text { B1 } & 0.40328(9) & 0.2462(2) & 0.50178(12) & 0.0300(3)\end{array}$

Atomic displacement parameters $\left(\AA^{2}\right)$

\begin{tabular}{lllllll}
\hline & $U^{11}$ & $U^{22}$ & $U^{33}$ & $U^{12}$ & $U^{13}$ & $U^{23}$ \\
\hline O1 & $0.0376(5)$ & $0.0461(5)$ & $0.0209(4)$ & $0.0093(4)$ & $0.0038(3)$ & $0.0008(3)$ \\
O2 & $0.0375(5)$ & $0.0500(5)$ & $0.0212(4)$ & $0.0078(4)$ & $0.0055(3)$ & $0.0041(4)$ \\
C1 & $0.0372(6)$ & $0.0315(6)$ & $0.0212(5)$ & $-0.0021(5)$ & $-0.0008(4)$ & $-0.0028(4)$ \\
C2 & $0.0476(7)$ & $0.0370(6)$ & $0.0274(6)$ & $-0.0065(5)$ & $0.0009(5)$ & $0.0002(5)$ \\
C3 & $0.0669(9)$ & $0.0306(6)$ & $0.0333(6)$ & $-0.0033(6)$ & $-0.0060(6)$ & $0.0046(5)$ \\
C4 & $0.0575(8)$ & $0.0333(6)$ & $0.0367(7)$ & $0.0098(6)$ & $-0.0093(6)$ & $-0.0037(5)$ \\
C5 & $0.0429(7)$ & $0.0357(6)$ & $0.0271(6)$ & $0.0062(5)$ & $-0.0058(5)$ & $-0.0069(5)$ \\
C6 & $0.0384(7)$ & $0.0532(8)$ & $0.0413(7)$ & $0.0139(6)$ & $-0.0034(5)$ & $-0.0098(6)$ \\
C7 & $0.0334(7)$ & $0.0664(9)$ & $0.0434(7)$ & $0.0036(6)$ & $0.0047(5)$ & $-0.0042(7)$ \\
C8 & $0.0368(6)$ & $0.0500(7)$ & $0.0366(7)$ & $-0.0038(6)$ & $0.0036(5)$ & $0.0016(6)$ \\
C9 & $0.0335(6)$ & $0.0348(6)$ & $0.0293(6)$ & $0.0010(5)$ & $-0.0007(4)$ & $-0.0010(5)$ \\
C10 & $0.0349(6)$ & $0.0311(6)$ & $0.0221(5)$ & $0.0009(5)$ & $-0.0022(4)$ & $-0.0051(4)$ \\
B1 & $0.0308(6)$ & $0.0366(7)$ & $0.0226(6)$ & $-0.0031(5)$ & $0.0024(4)$ & $-0.0013(5)$ \\
& & & & & & \\
\hline
\end{tabular}

Geometric parameters $\left(\AA,{ }^{\circ}\right)$

\begin{tabular}{llll}
\hline $\mathrm{O} 1-\mathrm{H} 1$ & $0.899(19)$ & $\mathrm{C} 4-\mathrm{C} 5$ & $1.4146(18)$ \\
$\mathrm{O} 1-\mathrm{B} 1$ & $1.3620(15)$ & $\mathrm{C} 5-\mathrm{C} 6$ & $1.4206(19)$ \\
$\mathrm{O} 2-\mathrm{H} 2$ & $0.886(19)$ & $\mathrm{C} 5-\mathrm{C} 10$ & $1.4243(16)$ \\
$\mathrm{O} 2-\mathrm{B} 1$ & $1.3706(14)$ & $\mathrm{C} 6-\mathrm{H} 6$ & $0.979(16)$ \\
$\mathrm{C} 1-\mathrm{C} 2$ & $1.3839(16)$ & $\mathrm{C} 6-\mathrm{C} 7$ & $1.361(2)$ \\
$\mathrm{C} 1-\mathrm{C} 10$ & $1.4382(16)$ & $\mathrm{C} 7-\mathrm{H} 7$ & $0.977(16)$ \\
$\mathrm{C} 1-\mathrm{B} 1$ & $1.5705(18)$ & $\mathrm{C} 7-\mathrm{C} 8$ & $1.4036(19)$ \\
$\mathrm{C} 2-\mathrm{H} 2 \mathrm{~A}$ & $0.971(15)$ & $\mathrm{C} 8-\mathrm{H} 8$ & $1.008(16)$ \\
$\mathrm{C} 2-\mathrm{C} 3$ & $1.4085(19)$ & $\mathrm{C} 8-\mathrm{C} 9$ & $1.3703(17)$ \\
$\mathrm{C} 3-\mathrm{H} 3$ & $0.983(16)$ & $\mathrm{C} 9-\mathrm{H} 9$ & $0.977(14)$ \\
$\mathrm{C} 3-\mathrm{C} 4$ & $1.362(2)$ & $\mathrm{C} 9-\mathrm{C} 10$ & $1.4180(16)$ \\
$\mathrm{C} 4-\mathrm{H} 4$ & $1.004(16)$ & & $120.98(12)$ \\
$\mathrm{B} 1-\mathrm{O} 1-\mathrm{H} 1$ & & & 119.5 \\
$\mathrm{~B} 1-\mathrm{O} 2-\mathrm{H} 2$ & $114.0(10)$ & $\mathrm{C} 7-\mathrm{C} 6-\mathrm{C} 5$ & 120.0 \\
$\mathrm{C} 2-\mathrm{C} 1-\mathrm{C} 10$ & $117.8(11)$ & $\mathrm{C} 7-\mathrm{C} 6-\mathrm{H} 6$ & $\mathrm{C} 6-\mathrm{C} 7-\mathrm{H} 7$ \\
\end{tabular}




\begin{tabular}{|c|c|c|c|}
\hline $\mathrm{C} 2-\mathrm{C} 1-\mathrm{B} 1$ & $118.27(11)$ & $\mathrm{C} 6-\mathrm{C} 7-\mathrm{C} 8$ & $119.95(12)$ \\
\hline $\mathrm{C} 10-\mathrm{C} 1-\mathrm{B} 1$ & $123.72(10)$ & $\mathrm{C} 8-\mathrm{C} 7-\mathrm{H} 7$ & 120.0 \\
\hline $\mathrm{C} 1-\mathrm{C} 2-\mathrm{H} 2 \mathrm{~A}$ & 118.9 & $\mathrm{C} 7-\mathrm{C} 8-\mathrm{H} 8$ & 119.7 \\
\hline $\mathrm{C} 1-\mathrm{C} 2-\mathrm{C} 3$ & $122.23(12)$ & $\mathrm{C} 9-\mathrm{C} 8-\mathrm{C} 7$ & $120.68(13)$ \\
\hline $\mathrm{C} 3-\mathrm{C} 2-\mathrm{H} 2 \mathrm{~A}$ & 118.9 & $\mathrm{C} 9-\mathrm{C} 8-\mathrm{H} 8$ & 119.7 \\
\hline $\mathrm{C} 2-\mathrm{C} 3-\mathrm{H} 3$ & 119.9 & $\mathrm{C} 8-\mathrm{C} 9-\mathrm{H} 9$ & 119.4 \\
\hline $\mathrm{C} 4-\mathrm{C} 3-\mathrm{C} 2$ & $120.12(12)$ & $\mathrm{C} 8-\mathrm{C} 9-\mathrm{C} 10$ & $121.23(11)$ \\
\hline $\mathrm{C} 4-\mathrm{C} 3-\mathrm{H} 3$ & 119.9 & $\mathrm{C} 10-\mathrm{C} 9-\mathrm{H} 9$ & 119.4 \\
\hline $\mathrm{C} 3-\mathrm{C} 4-\mathrm{H} 4$ & 119.7 & $\mathrm{C} 5-\mathrm{C} 10-\mathrm{C} 1$ & $119.49(11)$ \\
\hline $\mathrm{C} 3-\mathrm{C} 4-\mathrm{C} 5$ & $120.60(12)$ & $\mathrm{C} 9-\mathrm{C} 10-\mathrm{C} 1$ & $122.78(10)$ \\
\hline $\mathrm{C} 5-\mathrm{C} 4-\mathrm{H} 4$ & 119.7 & $\mathrm{C} 9-\mathrm{C} 10-\mathrm{C} 5$ & $117.72(11)$ \\
\hline $\mathrm{C} 4-\mathrm{C} 5-\mathrm{C} 6$ & $121.00(12)$ & $\mathrm{O} 1-\mathrm{B} 1-\mathrm{O} 2$ & $116.98(11)$ \\
\hline $\mathrm{C} 4-\mathrm{C} 5-\mathrm{C} 10$ & $119.56(12)$ & $\mathrm{O} 1-\mathrm{B} 1-\mathrm{C} 1$ & $121.32(10)$ \\
\hline $\mathrm{C} 6-\mathrm{C} 5-\mathrm{C} 10$ & $119.43(12)$ & $\mathrm{O} 2-\mathrm{B} 1-\mathrm{C} 1$ & $121.66(10)$ \\
\hline $\mathrm{C} 5-\mathrm{C} 6-\mathrm{H} 6$ & 119.5 & & \\
\hline $\mathrm{C} 1-\mathrm{C} 2-\mathrm{C} 3-\mathrm{C} 4$ & $-0.16(19)$ & $\mathrm{C} 6-\mathrm{C} 5-\mathrm{C} 10-\mathrm{C} 9$ & $-0.34(16)$ \\
\hline $\mathrm{C} 2-\mathrm{C} 1-\mathrm{C} 10-\mathrm{C} 5$ & $0.31(15)$ & $\mathrm{C} 6-\mathrm{C} 7-\mathrm{C} 8-\mathrm{C} 9$ & $-0.5(2)$ \\
\hline $\mathrm{C} 2-\mathrm{C} 1-\mathrm{C} 10-\mathrm{C} 9$ & $179.01(10)$ & $\mathrm{C} 7-\mathrm{C} 8-\mathrm{C} 9-\mathrm{C} 10$ & $0.45(18)$ \\
\hline $\mathrm{C} 2-\mathrm{C} 1-\mathrm{B} 1-\mathrm{O} 1$ & $139.56(12)$ & $\mathrm{C} 8-\mathrm{C} 9-\mathrm{C} 10-\mathrm{C} 1$ & $-178.73(11)$ \\
\hline $\mathrm{C} 2-\mathrm{C} 1-\mathrm{B} 1-\mathrm{O} 2$ & $-38.24(16)$ & $\mathrm{C} 8-\mathrm{C} 9-\mathrm{C} 10-\mathrm{C} 5$ & $-0.01(16)$ \\
\hline $\mathrm{C} 2-\mathrm{C} 3-\mathrm{C} 4-\mathrm{C} 5$ & $0.06(19)$ & $\mathrm{C} 10-\mathrm{C} 1-\mathrm{C} 2-\mathrm{C} 3$ & $-0.04(17)$ \\
\hline $\mathrm{C} 3-\mathrm{C} 4-\mathrm{C} 5-\mathrm{C} 6$ & $-178.59(12)$ & $\mathrm{C} 10-\mathrm{C} 1-\mathrm{B} 1-\mathrm{O} 1$ & $-41.91(16)$ \\
\hline $\mathrm{C} 3-\mathrm{C} 4-\mathrm{C} 5-\mathrm{C} 10$ & $0.22(18)$ & $\mathrm{C} 10-\mathrm{C} 1-\mathrm{B} 1-\mathrm{O} 2$ & $140.30(11)$ \\
\hline $\mathrm{C} 4-\mathrm{C} 5-\mathrm{C} 6-\mathrm{C} 7$ & $179.08(12)$ & $\mathrm{C} 10-\mathrm{C} 5-\mathrm{C} 6-\mathrm{C} 7$ & $0.27(18)$ \\
\hline $\mathrm{C} 4-\mathrm{C} 5-\mathrm{C} 10-\mathrm{C} 1$ & $-0.41(16)$ & $\mathrm{B} 1-\mathrm{C} 1-\mathrm{C} 2-\mathrm{C} 3$ & $178.58(11)$ \\
\hline $\mathrm{C} 4-\mathrm{C} 5-\mathrm{C} 10-\mathrm{C} 9$ & $-179.17(10)$ & $\mathrm{B} 1-\mathrm{C} 1-\mathrm{C} 10-\mathrm{C} 5$ & $-178.22(10)$ \\
\hline $\mathrm{C} 5-\mathrm{C} 6-\mathrm{C} 7-\mathrm{C} 8$ & $0.2(2)$ & $\mathrm{B} 1-\mathrm{C} 1-\mathrm{C} 10-\mathrm{C} 9$ & $0.47(16)$ \\
\hline $\mathrm{C} 6-\mathrm{C} 5-\mathrm{C} 10-\mathrm{C} 1$ & $178.42(10)$ & & \\
\hline
\end{tabular}

Hydrogen-bond geometry $\left(\AA,{ }^{\circ}\right)$

\begin{tabular}{lllll}
\hline$D-\mathrm{H}^{\cdots} A$ & $D-\mathrm{H}$ & $\mathrm{H} \cdots A$ & $D \cdots A$ & $D-\mathrm{H}^{\cdots} A A$ \\
\hline $\mathrm{O} 1-\mathrm{H} 1 \cdots \mathrm{O} 2^{\mathrm{i}}$ & $0.897(18)$ & $1.846(18)$ & $2.7411(13)$ & $176.3(17)$ \\
$\mathrm{O} 2-\mathrm{H} 2 \cdots \mathrm{O} 1^{\mathrm{ii}}$ & $0.888(19)$ & $1.891(19)$ & $2.7607(11)$ & $166.0(17)$ \\
$\mathrm{C} 9-\mathrm{H} 9 \cdots \mathrm{O} 1$ & $0.98(1)$ & $2.43(1)$ & $3.0911(15)$ & $124(1)$ \\
\hline
\end{tabular}

Symmetry codes: (i) $-x+1,-y,-z+1$; (ii) $x,-y+1 / 2, z-1 / 2$. 